06.3

\title{
Дважды отрицательные среды на основе антиферромагнитных метаматериалов для терагерцевого диапазона частот
}

\author{
() С.В. Гришин ${ }^{1}$, М.Д. Амельченко ${ }^{1}$, Ю.П. Шараевский ${ }^{1}$, С.А. Никитов ${ }^{2}$ \\ ${ }^{1}$ Саратовский национальный исследовательский государственный университет им. Н.Г. Чернышевского, Саратов, Россия \\ ${ }^{2}$ Институт радиотехники и электроники им. В.А. Котельникова РАН, Москва, Россия \\ E-mail: sergrsh@yandex.ru
}

Поступило в Редакцию 17 мая 2021г.

В окончательной редакции 7 июня 2021 г.

Принято к публикации 8 июня 2021 г.

\begin{abstract}
Приведены результаты теоретического исследования дисперсионных характеристик электромагнитных волн, существующих в антиферромагнитном (АФМ) метаматериале. АФМ-метаматериал состоит из поперечно намагниченного антиферромагнетика, внутри которого находится двумерная периодическая структура из тонких проводящих проволок, окруженных изоляторами. Установлено, что АФМ-метаматериал обладает двумя областями частот, в которых существуют обратные волны, а материальные параметры среды являются дважды отрицательными. Указанные области находятся в терагерцевом диапазоне.
\end{abstract}

Ключевые слова: метаматериалы, левые среды, антиферромагнетики, спиновые волны.

DOI: 10.21883/PJTF.2021.18.51470.18873

Известно, что метаматериалы представляют собой искусственно созданные среды со свойствами, не встречающимися у обычных природных сред [1]. Среди метаматериалов особый класс составляют дважды отрицательные среды (или ,левые“ среды), у которых диэлектрическая $\varepsilon$ и магнитная $\mu$ проницаемости являются одновременно отрицательными величинами [2]. Это приводит к появлению у среды отрицательного показателя преломления и распространению в ней обратной электромагнитной волны (ЭМВ).

Начиная с 2000-х годов как у нас в стране [3,4], так и за рубежом [5-9] стала развиваться концепция перестраиваемых магнитным полем дважды отрицательных сред. Для их создания использовались ферромагнитные материалы. Это были либо пленки $[4,6]$ и пластины [7] железо-иттриевого граната, либо ферритовые стержни [8] или пленки ВаM ферритов и гексаферритов [9], работающие в микроволновом диапазоне. Как известно [10], ферромагнетики (ФМ) относятся к $\mu$-отрицательным средам, у которых магнитная проницаемость принимает отрицательные значения в определенном интервале частот, находящемся в микроволновом диапазоне. Совмещение их свойств со свойствами $\varepsilon$-отрицательных сред, в качестве которых использовались периодические решетки из тонких проволок, давало возможность реализовать дважды отрицательные среды без использования дополнительных субволновых элементов в виде кольцевых разомкнутых резонаторов.

Помимо создания дважды отрицательных сред на основе ФМ, работающих в микроволновом диапазоне, были предприняты попытки создать аналогичные среды и функциональные устройства для терагерцевого диапазона [1,11-13]. Так, в [14] было проведено численное моделирование показателя преломления метаматериала, выполненного на основе ферритовых пленок LuBiIG и серебряных полосок, который имел отрицательные значения на частотах выше $100 \mathrm{GHz}$. Была продемонстрирована перестройка полосы частот данного метаматериала внешним постоянным магнитным полем. Другим магнитным материалом, работающим в терагерцевой области, является антиферромагнетик (АФМ) [15-17]. В отличие от ФМ кристаллическая решетка АФМ состоит из двух магнитных подрешеток. Это обусловливает наличие у АФМ четырех характерных частот, на которых магнитная проницаемость меняет свой знак. Таким образом, целью настоящей работы является создание на основе АФМ дважды отрицательного метаматериала с двумя полосами частот обратных ЭМВ, находящимися в терагерцевом диапазоне.

Анализируемая структура представляет собой безграничный АФМ, в котором находится двумерная (2D) периодическая структура из тонких проволок с периодом $a$. Предполагается, что период структуры намного меньше длины ЭМВ $\lambda$, т.е. $a \ll \lambda$. Исследуется случай поперечного намагничивания, когда внешнее постоянное магнитное поле $\mathbf{H}_{0}$ направлено вдоль проволок, а волновой вектор $\mathbf{k}$ направлен перпендикулярно магнитному полю $\mathbf{H}_{0}\left(\mathbf{k} \perp \mathbf{H}_{0}\right)$. При таком виде намагничивания в магнетике существуют ЭМВ с линейной поляризацией [10]. В этом случае электрическое поле ЭМВ направлено вдоль проволок, а магнитное поле ЭМВ является ортогональным электрическому полю и волновому вектору $\mathbf{k}$. Схематическое изображение данной структуры приведено на рис. 1, $a$.

В [5] было показано, что для получения дважды отрицательной среды из магнитного материала необходимо окружить проводящие проволоки, находящиеся внутри магнитной матрицы, немагнитным диэлектриком. 

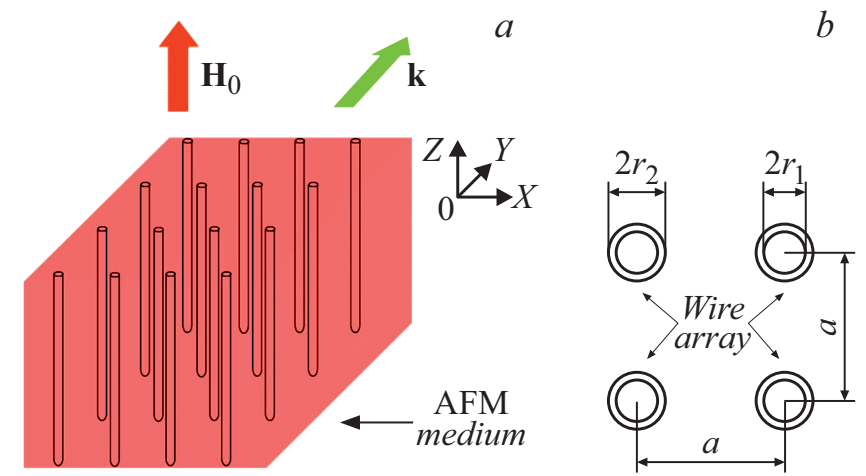

Рис. 1. Схематические изображения поперечно намагниченного безграничного АФМ-метаматериала $(a)$ и $2 \mathrm{D}$-периодической структуры (вид сверху) из тонких проволок радиусом $r_{1}$, окруженных немагнитным изолятором радиусом $r_{2}(b)$.

Радиус проволоки $r_{1}$ выбирался намного меньшим, чем период структуры $a$, а внешний радиус изолирующей оболочки $r_{2}$ выбирался из условий, что $r_{2} \cong\left(r_{1} a\right)^{1 / 2}$ и $r_{1} \ll r_{2} \ll a$ (рис. $\left.1, b\right)$.

Известно [10], что тензор высокочастотной магнитной

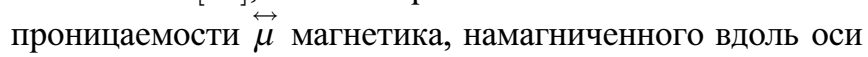
$0 Z\left(\mathbf{H}_{0} \| \mathbf{0 Z}\right)$, задается как

$$
\overleftrightarrow{\mu}=\left(\begin{array}{ccc}
\mu & j \mu_{a} & 0 \\
-j \mu_{a} & \mu & 0 \\
0 & 0 & 1
\end{array}\right)
$$

где диагональные $\mu$ и недиагональные $\mu_{a}$ компоненты тензора для АФМ с „легкой“ осью анизотропии, совпадающей с осью $0 Z$, запишутся в следующем виде [17]:

$$
\begin{gathered}
\mu=1+8 \pi \gamma_{s}^{2} M_{s} H_{A}\left(\omega_{+} \omega_{-}-\omega^{2}\right) /\left[\left(\omega_{+}^{2}-\omega^{2}\right)\left(\omega_{-}^{2}-\omega^{2}\right)\right], \\
\mu_{a}=8 \pi \gamma_{s}^{2} M_{s} H_{A} \omega\left(\omega_{-}-\omega_{+}\right) /\left[\left(\omega_{+}^{2}-\omega^{2}\right)\left(\omega_{-}^{2}-\omega^{2}\right)\right],
\end{gathered}
$$

где $\gamma_{s}$ - усредненный $g$-фактор, $M_{s}-$ усредненная статическая намагиченность подрешеток, $H_{A}-$ поле анизотропии, $\omega_{+}=\gamma_{s}\left(H_{C}+H_{0}\right), \omega_{-}=\gamma_{s}\left(H_{C}-H_{0}\right)-$ частоты антиферромагнитного резонанса, $H_{C}=\left[H_{A}\left(2 H_{E}+H_{A}\right)\right]^{1 / 2}-$ поле „опрокидывания“ подрешеток, $H_{E}-$ поле однородного обменного взаимодействия между подрешетками.

При решении электродинамической задачи в приближении однородных плоских волн для поперечно намагниченного безграничного АФМ-метаматериала, получаем дисперсионное уравнение в виде

$$
k=k_{0}\left(\mu_{e f f \perp} \varepsilon_{e f f \perp}\right)^{1 / 2},
$$

где $k$ - волновое число ЭМВ в среде, $k_{0}=\omega / c-$ волновое число ЭМВ в вакууме, $\omega=2 \pi f-$ круговая

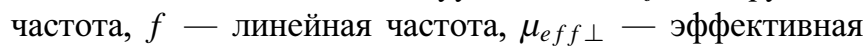
магнитная проницаемость поперечно намагниченного магнетика, которая определяется на основе следующего выражения [10]:

$$
\mu_{e f f \perp}=\left(\mu^{2}-\mu_{a}^{2}\right) / \mu,
$$

$\varepsilon_{e f f \perp}-$ эффективная диэлектрическая проницаемость поперечно намагниченного магнетика, которая в [5] имела следующий вид:

$$
\begin{gathered}
\varepsilon_{e f f \perp}=\varepsilon_{r}\left[1-\omega_{p \perp}^{2} /\left(\omega^{2}+i \alpha_{\perp}\right)\right], \\
\omega_{p \perp}^{2} \cong 2 \pi /\left\langle\varepsilon _ { f } a ^ { 2 } \mu _ { 0 } \left\{\ln \left(r_{2} / r_{1}\right)+\mu_{e f f \perp}\left[\ln \left(a / r_{2}\right)\right.\right.\right. \\
-(3+\ln 2-\pi / 2) / 2]\}\rangle,
\end{gathered}
$$

$\alpha_{\perp}=\varepsilon_{f} \omega \omega_{p \perp}^{2} / \sigma_{e f f}, \varepsilon_{f}=\varepsilon_{0} \varepsilon_{r}-$ абсолютная диэлектрическая проницаемость магнетика, $\varepsilon_{0}=1 /\left(\mu_{0} c^{2}\right)$ - электрическая постоянная, $\mu_{0}$ - магнитная постоянная, $\varepsilon_{r}-$ относительная диэлектрическая проницаемость магнетика, $\sigma_{e f f}=\pi r_{1}^{2} \sigma / a^{2}-$ эффективная проводимость проволочной структуры, $\sigma$ - электрическая проводимость проволоки. Выражение (5) было получено в предположении однородности плотности тока, протекающего через проволоку. Данное предположение выполняется, когда радиус проволоки намного меньше глубины скин-слоя $\delta$, т. е. $r_{1} \ll \delta=\left(2 / \mu_{0} \sigma \omega\right)^{1 / 2}[5]$.

Условия, при которых $\mu_{e f f \perp}<0$, для АФМ запишутся как

$$
\begin{aligned}
& \omega_{\perp 1}<\omega<\omega_{\text {ar } 1}, \\
& \omega_{\perp 2}<\omega<\omega_{\text {ar } 2},
\end{aligned}
$$

где $\omega_{\perp 1,2}=\left[ \pm\left(\omega_{+}-\omega_{-}\right)+D_{1}^{1 / 2}\right] / 2-$ две частоты АФМ-резонанса при поперечном намагничивании, $\omega_{a r 1,2}=\left[\left(\omega_{+}^{2}+\omega_{-}^{2}+8 \pi \gamma_{s}^{2} M_{s} H_{A} \pm D_{2}^{1 / 2}\right) / 2\right]^{1 / 2}$ - две частоты АФМ-антирезонанса,

$$
\begin{aligned}
D_{1} & =\left(\omega_{+}+\omega_{-}\right)^{2}+32 \pi \gamma_{s}^{2} M_{s} H_{A}, \\
D_{2} & =\left(\omega_{+}^{2}+\omega_{-}^{2}+8 \pi \gamma_{s}^{2} M_{s} H_{A}\right)^{2} \\
& -4 \omega_{+} \omega_{-}\left(\omega_{+} \omega_{-}+8 \pi \gamma_{s}^{2} M_{s} H_{A}\right) .
\end{aligned}
$$

Предлагаемая к рассмотрению модель учитывает только временну́ю дисперсию среды. Она не учитывает пространственную дисперсию проволочной структуры [18], а также отражение ЭМВ от периодической структуры, как это делалось в [5].

На рис. 2, $a, b$ приведены дисперсионные характеристики (ДХ) ЭМВ (сплошные линии), которые рассчитаны на основе (3) с учетом (4)-(6) и (2). На рис. $2, c, d$ приведены частотные зависимости эффективных материальных параметров среды. Из представленных на рис. 2, $a, c$ результатов следует, что в отсутствие $2 \mathrm{D}$-периодической структуры $\left(\omega_{p \perp}=0\right)$ в АФМ существует три необыкновенные ЭМВ. Две из них являются медленными ЭМВ (кривые 2 и 3), а третья - быстрой ЭМВ (кривая 1). Все три ЭМВ находятся на частотах, где $\mu_{e f f \perp}>0$. Частота отсечки быстрой ЭМВ определяется частотой $\omega_{a r 1}$. У низкочастотной медленной ЭМВ частота отсечки отсутствует, а у высокочастотной медленной ЭМВ частота отсечки соответствует $\omega_{a r 2}$. Обе медленные ЭМВ характеризуются предельными частотами $\omega_{\perp 1,2}$, находящимися в терагерцевом диапазоне. 

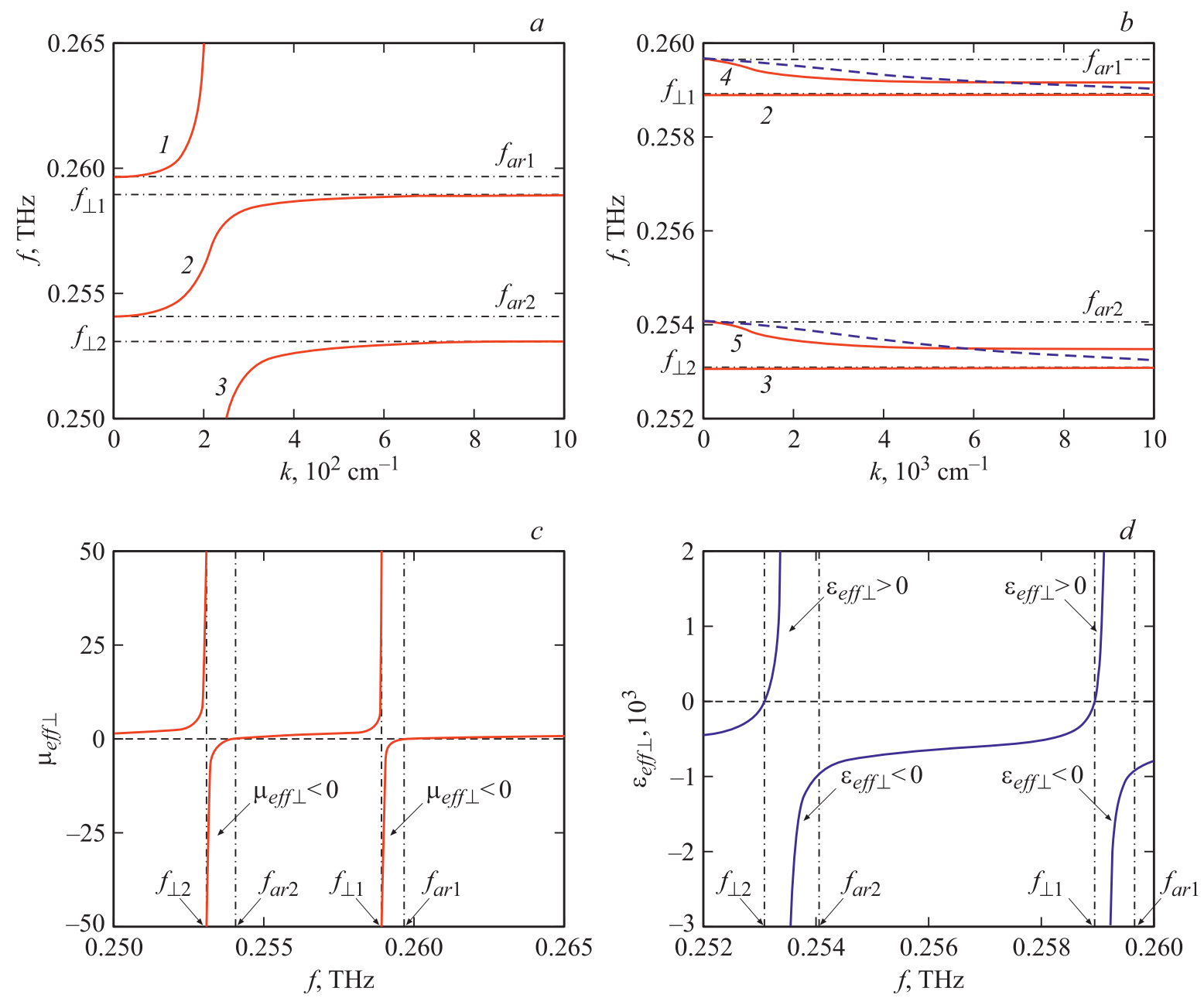

Рис. 2. Дисперсионные характеристики необыкновенных быстрой (1) и медленных (кривые $2-5)$ ЭМВ $(a, b)$, а также частотные зависимости $\mu_{e f f \perp}(c)$ и $\varepsilon_{e f f \perp}(d)$, рассчитанные для поперечно намагниченных АФМ $(a, c)$ и АФМ-метаматериала $(b, d)$. На части $b$ штриховыми линиями показаны ДХ обратных ЭМВ, рассчитанные для поперечного намагниченного АФМ-метаматериала с эффективной диэлектрической проницаемостью, соответствующей изотропной плазме, $-\varepsilon_{e f f}=\varepsilon_{r}\left(1-\omega_{p}^{2} / \omega^{2}\right)$, где $\omega_{p}^{2}=2 \pi c^{2} /\left[a^{2} \ln \left(a / r_{1}\right)\right]$. Расчеты выполнены для $\omega_{p \perp}=0(a, c)$ и для $a=10^{-3} \mathrm{~cm}, r_{1}=10^{-5} \mathrm{~cm}, r_{2}=10^{-4} \mathrm{~cm}(b, d)$. Во всех случаях $H_{0}=79.58 \mathrm{kA} / \mathrm{m}, M_{s}=0.0560 \mathrm{~T}, H_{E}=40.98 \mathrm{MA} / \mathrm{m}, H_{A}=636.64 \mathrm{kA} / \mathrm{m}$ и $\varepsilon_{r}=16$. Параметры АФМ соответствовали $\mathrm{MnF}_{2}[17]$.

Таким образом, медленные ЭМВ обладают нормальной дисперсией.

На рис. 2, $b$ приведены ДХ ЭМВ, полученные при наличии 2D-периодической структуры из идеально проводящих проволок $\left(\omega_{p \perp} \neq 0\right.$ и $\left.\alpha_{\perp}=0\right)$, радиус которых меньше глубины скин-слоя меди на частоте $0.3 \mathrm{THz}$. Видно, что в этом случае наблюдается вырождение медленных прямых ЭМВ (кривые 2, 3) в колебания на частотах $\omega_{\perp 1,2}$, так как в полосе частот существования этих волн $\mu_{e f f \perp}>0$, а $\varepsilon_{e f f \perp}<0$ (рис. 2, $c, d$ ). Частота отсечки быстрой ЭМВ перестает соответствовать частоте $\omega_{a r 1}$ и смещается в более высокочастотную область $\left(>1.5 \mathrm{THz}\right.$ ), где $\mu_{e f f \perp}>0$ и $\varepsilon_{e f f \perp}>0$ (рис. 2, $\left.c, d\right)$. Наиболее значимый результат заключается в появлении двух обратных медленных ЭМВ (кривые 4, 5), находящихся в разных частотных диапазонах, где $\mu_{e f f \perp}<0$ и $\varepsilon_{e f f \perp}<0$ (рис. $2, c, d$ ). Необходимо отметить, что на частотах, где $\mu_{e f f \perp}<0$, эффективная диэлектрическая проницаемость среды может быть как меньше, так и больше нуля. В связи с этим каждая обратная ЭМВ занимает

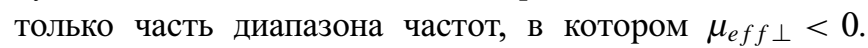
На рис. 2, $b$ приведены также ДХ двух обратных ЭМВ (штриховые линии), рассчитанные на основе (2)-(4) для АФМ-метаматериала с эффективной диэлектрической проницаемостью, соответствующей изотропной плазме (см. выражение, приведенное в подписи к рис. 2) [19]. Видно, что в данном приближении (изоляторы имеют бесконечно большой радиус) обратные ЭМВ являются более замедленными и занимают более широкую полосу частот, которая определяется выражениями (7a) и (7b).

В заключение отметим, что полученные результаты представляют интерес для разработки функциональных материалов для терагерцевой магноники. 


\section{Финансирование работы}

Работа выполнена за счет гранта Российского научного фонда (проект № 19-79-20121).

\section{Конфликт интересов}

Авторы заявляют, что у них нет конфликта интересов.

\section{Список литературы}

[1] Metamaterials. Devices and applications, ed. by A.L. Borja (InTech Open, 2017). DOI: 10.5772/67198

[2] В.Г. Веселаго, УФН, 92 (3), 517 (1967). DOI: $10.3367 /$ UFNr.0092.196707d.0517

[3] Ю.И. Беспятых, А.С. Бугаев, И.Е. Дикштейн, ФТТ, 43 (11), 2043 (2001).

[4] А.В. Вашковский, Э.Г. Локк, УФН, 174 (6), 657 (2004). DOI: $10.3367 /$ UFNr.0174.200406e.0657

[5] G. Dewar, New J. Phys., 7 (1), 161 (2005). DOI: $10.1088 / 1367-2630 / 7 / 1 / 161$

[6] Y. He, P. He, S.D. Yoon, P.V. Parimic, F.J. Rachfordd, V.G. Harris, C. Vittoria, J. Magn. Magn. Mater., 313 (1), 187 (2007). DOI: 10.1016/j.jmmm.2006.12.031

[7] H. Zhao, J. Zhou, Q. Zhao, B. Li, L. Kang, Y. Bai, Appl. Phys. Lett., 91 (13), 131107 (2007). DOI: 10.1063/1.2790500

[8] K. Bi, J. Zhou, H. Zhao, X. Liu, C. Lan, Opt. Express, 21 (9), 10746 (2013). DOI: 10.1364/OE.21.010746

[9] F.J. Rachford, D.N. Armstead, V.G. Harris, C. Vittoria, Phys. Rev. Lett., 99 (5), 057202 (2007).

[10] А.Г. Гуревич, Ферриты на сверхвысоких частотах (Физматлит, М., 1960).

[11] A.B. Ustinov, G. Srinivasan, Appl. Phys. Lett., 93 (14), 142503 (2008). DOI: 10.1063/1.2996585

[12] F. Fan, S.J. Chang, C. Niu, Y. Hou, X.-H. Wang, Opt. Commun., 285 (18), 3763 (2012). DOI: $10.1016 /$ j.optcom.2012.05.044

[13] M. Białek, T. Ito, H.M. Ronnow, J.-P. Ansermet, Phys. Rev. B, 99 (6), 064429 (2019). DOI: 10.1103/PhysRevB.99.064429

[14] Y.J. Huang, G.J. Wen, T.Q. Li, J.L.W. Li, K. Xie, IEEE Antennas Wireless Propagat. Lett., 11, 264 (2012). DOI: 10.1109/LAWP.2012.2189090

[15] С.А. Никитов, А.Р. Сафин, Д.В. Калябин, А.В. Садовников, Е.Н. Бегинин, М.В. Логунов, М.А. Морозова, С.А. Одинцов, С.А. Осокин, А.Ю. Шараевская, Ю.П. Шараевский, А.И. Кирилюк, УФН, 190 (10), 1009 (2020). DOI: $10.3367 /$ UFNe.2019.07.038609

[16] А.Р. Сафин, С.А. Никитов, А.И. Кирилюк, Д.В. Калябин, А.В. Садовников, П.А. Стремоухов, М.В. Логунов, П.А. Попов, ЖЭТФ, 158 (1), 85 (2020). DOI: $10.31857 / \mathrm{S} 0044451020070081$

[17] A.Yu. Sharaevskaya, D.V. Kalyabin, E.N. Beginin, Y.K. Fetisov, S.A. Nikitov, J. Magn. Magn. Mater., 475, 778 (2019). DOI: 10.1016/j.jmmm.2018.11.130

[18] P.A. Belov, R. Marqués, S.I. Maslovski, I.S. Nefedov, M. Silveirinha, C.R. Simovski, S.A. Tretyakov, Phys. Rev. B, 67 (11), 113103 (2003). DOI: 10.1103/PhysRevB.67.113103

[19] М.Д. Амельченко, С.В. Гришин, Ю.П. Шараевский, Письма в ЖТФ, 45 (23), 14 (2019).

DOI: $10.21883 /$ PJTF.2019.23.48712.17830 